BMJ Open

Diabetes

Research

\& Care

\title{
Fetuin-A and fetal growth in gestational diabetes mellitus
}

Wen-Juan Wang, ${ }^{1,2}$ Lin Zhang, ${ }^{3}$ Tao Zheng, ${ }^{3}$ Guang-Hui Zhang, ${ }^{4}$ Kun Du, ${ }^{4}$ Meng-Nan Yang, ${ }^{1,3}$ Hua He, ${ }^{1,5}$ Shufan Wang, ${ }^{2}$ Weiye Wang, ${ }^{1}$ Jun Zhang, ${ }^{1}$ Fengxiu Ouyang, ${ }^{1}$ Zhong-Cheng Luo $\mathbb{1}\left(1,{ }^{1,2}\right.$ On behalf of the Shanghai Birth Cohort

To cite: Wang W-J, Zhang L, Zheng T, et al. Fetuin-A and fetal growth in gestational diabetes mellitus. BMJ Open Diab Res Care 2020;8:e000864. doi:10.1136/ bmjdrc-2019-000864

W-JW and LZ contributed equally.

This work was presented in abstract form at the Developmental Origins of Health and Disease (DOHaD) 2019 World Congress in Melbourne, Australia, 20-23 October 2019.

Received 29 August 2019 Revised 16 December 2019 Accepted 26 December 2019

A) Check for updates

(C) Author(s) (or their employer(s)) 2020. Re-use permitted under CC BY-NC. No commercial re-use. See rights and permissions. Published by BMJ.

For numbered affiliations see end of article.

Correspondence to Dr Zhong-Cheng Luo; zcluo@lunenfeld.ca

Dr Fengxiu Ouyang; ouyangfengxiu@xinhuamed. com.cn

\section{ABSTRACT}

Objective Fetuin-A is a glycoprotein produced by hepatocytes and has been associated with insulin resistance and bone growth in postnatal life. Gestational diabetes mellitus (GDM) is a condition characterized by insulin resistance. It is unclear whether GDM may affect cord blood fetuin-A levels and whether fetuin- $A$ is associated with fetal growth.

Research design and methods In a nested case-control study of 153 matched pairs of neonates of mothers with GDM and euglycemic pregnancies in the Shanghai Birth Cohort, we evaluated cord blood fetuin-A in association with GDM and fetal growth.

Results Comparing the newborns of GDM versus euglycemic mothers, cord blood fetuin-A concentrations were similar (mean \pm SD: $783.6 \pm 320.0$ vs $754.8 \pm 281.9 \mu \mathrm{g} /$ $\mathrm{mL}, \mathrm{p}=0.53$ ), while insulin-like growth factor (IGF)-I $(76.6 \pm 27.8 \mathrm{ng} / \mathrm{mL}$ vs $68.1 \pm 25.1 \mathrm{ng} / \mathrm{mL}, \mathrm{p}=0.008)$ and IGF-II $(195.3 \pm 32.5 \mathrm{ng} / \mathrm{mL}$ vs $187.5 \pm 30.8 \mathrm{ng} /$ $\mathrm{mL}, \mathrm{p}=0.042$ ) concentrations were higher. Cord blood fetuin-A was not correlated with insulin, IGF-I or IGF-II. Cord blood fetuin-A was negatively correlated with birth weight $(r=-0.19, p=0.025)$ and birth length $(r=-0.24$, $\mathrm{p}=0.005) \mathrm{z}$ scores in GDM pregnancies, while there were no significant correlations in euglycemic pregnancies (tests for interaction: $p=0.014$ for birth length, $p=0.013$ for birth length). Adjusting for maternal and neonatal characteristics, the differential associations remained. Conclusions GDM was not associated with cord blood fetuin-A levels. Fetuin-A was negatively associated with fetal growth in GDM but not in euglycemic pregnancies. This novel observation suggests a GDM-conditional negative correlation of fetuin-A with fetal growth.

\section{INTRODUCTION}

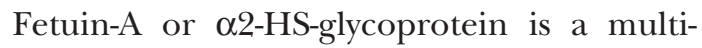
functional glycoprotein produced mainly by hepatocytes and is involved in a diverse range of physiological and pathological conditions. It has been linked to insulin signaling pathways, and may be involved in the development of insulin resistance and type two diabetes. ${ }^{1-3}$ Fetuin-A inhibits insulin receptor signaling $^{45}$ and is an endogenous ligand of toll-like receptor 4 promoting lipid-induced insulin resistance. ${ }^{6}$ Gestation diabetes mellitus (GDM), a condition characterized by elevated insulin resistance and impaired

\section{Significance of this study}

What is already known about this subject?

- Fetuin-A has been associated with insulin resistance and bone growth in postnatal life.

- Gestational diabetes mellitus (GDM) is a condition characterized by insulin resistance, it is unclear whether GDM may affect cord blood fetuin-A levels and whether fetuin-A is associated with fetal growth.

What are the new findings?

- GDM was not associated with cord blood fetuin-A levels in Chinese newborns.

- Fetuin-A was negatively correlated to fetal growth (birth weight or length $\mathrm{z}$ score) in GDM but not in euglycemic pregnancies, suggesting a GDMconditional negative correlation of fetuin-A with fetal growth.

\section{How might these results change the focus of} research or clinical practice?

- The GDM-associated intrauterine endocrine environment may enable a negative impact of fetuin-A on fetal growth. More studies are warranted to validate the finding in other populations.

glucose tolerance in the second half of the pregnancy, ${ }^{7}$ has been associated with elevated maternal circulating fetuin-A levels in some but not all studies. ${ }^{8-10}$ There is a lack of data on whether there are alterations in fetal/cord blood fetuin-A concentrations in GDM.

GDM is often associated with increased fetal size, which may be mainly attributable to elevated glucose transfer from the mother to the fetus. ${ }^{11}$ Fetuin-A expression is relatively high in the fetus and has been linked to postnatal bone growth. ${ }^{12-16}$ Animal studies in fetuin-A knockout mice have reported impaired growth plate chondrocyte maturation and stunted longitudinal femur growth, ${ }^{15} 17$ suggesting that fetuin-A is important for linear growth. In contrast, a clinical study has reported a negative association between maternal blood fetuin-A and birth length. ${ }^{9}$ We are unaware of any data on cord blood fetuin-A in relation to fetal growth. The 
relatively high concentrations of fetuin-A in the fetuses (compared with the adults) raise the possibility that it may play an important role during fetal development. ${ }^{12} 14$ It remains unknown whether cord blood fetuin-A is associated with fetal growth and whether GDM may modify the association.

In view of the above-discussed knowledge gaps, we sought to determine whether cord blood fetuin-A levels are different in GDM versus euglycemic pregnancies, and to assess whether cord blood fetuin-A levels are associated with fetal growth in pregnancies with or without GDM. We hypothesized that GDM may affect cord blood fetuin-A levels and may modify the association between fetuin-A and fetal growth since GDM may cause numerous changes in the intrauterine endocrine environment.

\section{RESEARCH DESIGN AND METHODS}

\section{Study design, data and specimens}

We conducted a nested matched (1:1) case-control study based on the recently described Shanghai Birth Cohort (SBC) ${ }^{18}$ Briefly, the SBC was built to examine the effects of perinatal factors on birth outcomes, infant growth and development, including 4127 pregnant women recruited in six urban obstetric care centers in Shanghai, 2013-2016. ${ }^{18}$

GDM was diagnosed according to the International Association of Diabetes and Pregnancy Study Group (IADPSG) criteria $^{19}$; if any one of the glucose values was at or above the following thresholds in the $75 \mathrm{~g}$ oral glucose tolerance test (OGTT) at 24-28 weeks of gestation: fasting, $5.1 \mathrm{mmol} / \mathrm{L} ; 1$ hour, $1 . \mathrm{mmol} / \mathrm{L}$; and 2 hours, $8.5 \mathrm{mmol} / \mathrm{L}$. Cases were newborns of GDM mothers, and controls were newborns of mothers without diabetes. All case and controls were from the SBC cohort and must meet the following eligibility criteria: (1) Han ethnicity (the majority ethnic group, $>98 \%$ ); (2) maternal age of 20-45 years; (3) natural conception; (4) singleton pregnancy; (5) the mother was free of prepregnancy severe illnesses (eg, type 1 or type 2 diabetes and chronic hypertension) and life-threatening pregnancy complications (eg, pre-eclampsia/eclampsia); (6) the infant was free of birth defects; (7) 5 min Apgar score $\geq 7$; and (8) cord blood and placenta specimens available for future assays. All eligible newborns of GDM mothers $(n=153)$ were included. Controls were randomly sampled among all eligible subjects and matched to cases $(1: 1)$ by infant sex (the same) and gestational age (within 1week). Therefore, a total of 306 subjects (153 newborns of GDM mothers and 153 controls) constituted the study sample. Figure 1 presents the flowchart in the selection of study subjects.

Birth weight was measured to the nearest $1 \mathrm{~g}$ and was transformed to z score using the 2015 Chinese sex-specific and gestational age-specific birthweight standards. ${ }^{20}$ Birth length was measured to the nearest $0.1 \mathrm{~cm}$ and was transformed to z score according to sex-specific and gestational age-specific means and SDs of all singleton infants in the SBC cohort since there are no Chinese

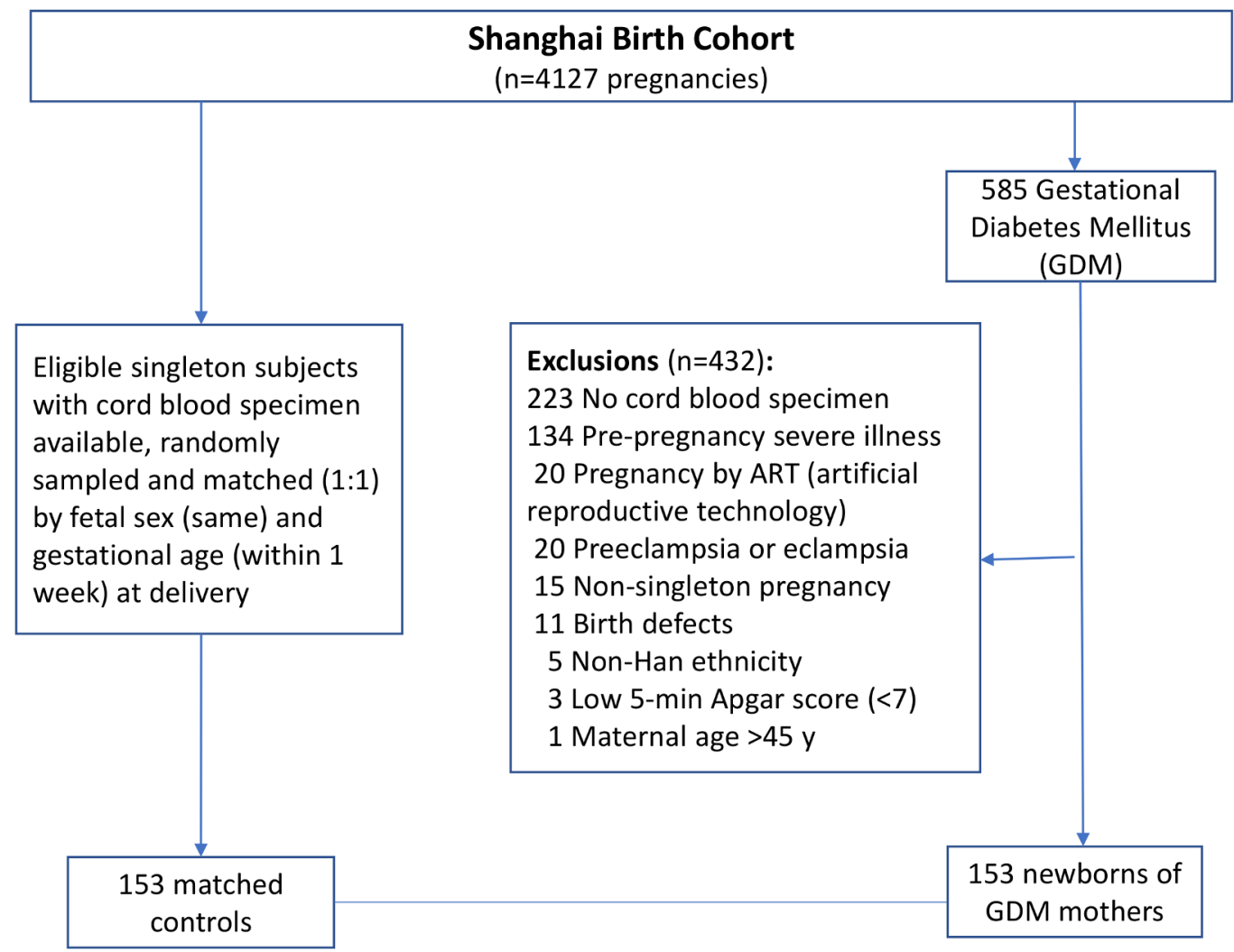

Figure 1 Flowchart in the selection of study subjects in a nested matched case-control study of the newborns of GDM mothers and controls in the Shanghai Birth Cohort. GDM, gestational diabetes mellitus. 
birth length standards available. Maternal prepregnancy Body Mass Index (BMI) $\left(\mathrm{kg} / \mathrm{m}^{2}\right)$ was categorized by Chinese standards: underweight $(<18.5)$, normal weight (18.5-23.9), overweight $(24.0-27.9)$ and obese $(\geq 28) .{ }^{21}$ Gestational weight gain $(\mathrm{kg})$ was calculated as the difference between the weight before delivery (within 1 week, measured by a clinic nurse using a routinely calibrated electronic weighing device) and prepregnancy weight (self-reported in the first antenatal care visit at 8-14 weeks of gestation), and standardized to $\mathrm{z}$ score according to the BMI category-specific mean and SD of gestational weight gain in the SBC.

Umbilical cord blood samples (in multiple tubes for serum (non-coagulant) and plasma (EDTA)) were collected after the clamping of the umbilical cord immediately after safe delivery of the baby. The cord blood samples were centrifuged in a centrifuge (Beckman Coulter Allegra X-15R, USA) at $4^{\circ} \mathrm{C}, 4000 \mathrm{r} / \mathrm{min}$ for $10 \mathrm{~min}$. The separated serum and plasma samples were stored at $-80^{\circ} \mathrm{C}$ until assays.

\section{Biochemical assays}

Cord plasma fetuin-A was measured by an ELISA kit (R\&D System, Minnesota, USA), and the absorbance was determined using a microplate spectrophotometer (Beckman CX7, USA). Cord serum insulin and insulinlike growth factor (IGF)-I concentrations were detected by automated chemiluminescent assays (ADVIA Centaur and Immulite 2000, Siemens, Germany). Cord plasma IGF-II was measured by an ELISA kit (R\&D System). The minimal detectable concentrations were $0.62 \mathrm{ng} /$ $\mathrm{mL}$ for fetuin-A, $3.5 \mathrm{pmol} / \mathrm{L}$ for insulin, $25 \mathrm{ng} / \mathrm{mL}$ for IGF-I, and $1.88 \mathrm{pg} / \mathrm{mL}$ for IGF-II, respectively. The intraassay and interassay coefficients of variation were in the ranges of $0.5 \%-8.3 \%$ for fetuin-A, $2.0 \%-6.5 \%$ for insulin and IGF-I, and $2.4 \%-9.3 \%$ for IGF-II, respectively. In all biomarker assays, the lab technicians were blinded to the clinical status (GDM?) of study subjects. Except for IGF-I, all biomarker values were above the detection limits. For IGF-I values below the detection limit $(n=16,5.2 \%)$, they were treated as missing in data analysis.

\section{Statistical analysis}

Mean \pm SD and median (IQR) were presented for continuous variables, while number $(\%)$ was presented for categorical variables. Pearson partial correlation coefficients were calculated to evaluate the associations between cord blood fetuin-A and fetal growth factors adjusting for gestational age at blood sampling. Generalized linear models were applied to assess the associations of fetuin-A with fetal growth (birth length/weight $\mathrm{z}$ scores) adjusted for maternal and neonatal characteristics. Biomarker data were log-transformed in correlation and regression analyses. All data analyses were performed using Statistical Analysis System, V.9.4. A p value of $<0.05$ was considered statistically significant for the primary research question on the difference in cord blood fetuin-A concentration between the newborns of GDM and controls. A p value of
$<0.025$ was considered statistically significant in the tests for interaction of GDM status with fetuin-A in relation to fetal growth (birthweight and birth length z scores, Bonferroni correction for two tests) by adding an interaction item in generalized linear models.

\section{RESULTS}

Table 1 presents maternal and neonatal characteristics of the study population. Family history of diabetes tended to be more common in women with GDM $(16.5 \%)$ versus euglycemic $(9.5 \%)$ pregnancies $(\mathrm{p}=0.056)$. Caesarean deliveries were more frequent in GDM versus euglycemic women $(54.3 \%$ vs $33.1 \%, \mathrm{p}<0.001)$. In the GDM group, $40.3 \%$ women were overweight/obese, much higher than that of $18.0 \%$ in euglycemic pregnancies. Gestational weight gains (in absolute value or z score) were lower in GDM versus euglycemic pregnancies. As expected, maternal glucose levels in the $75 \mathrm{~g}$ OGTT at 24-28 weeks of gestation were substantially higher in GDM versus euglycemic women (all $\mathrm{p}<0.001)$. There were 13 patients with GDM requiring the use of insulin in the management of hyperglycemia; other patients with GDM were treated with dietary and lifestyle interventions only. The newborns of mothers with GDM had higher birthweight $\mathrm{z}$ scores than the newborns of euglycemic mothers $(0.40 \pm 1.16$ vs $0.15 \pm 0.98, \mathrm{p}=0.044)$, while there were no significant differences in birth length (in centimetre or z score). On average, infants of mothers with GDM were delivered about 1 day ( 0.19 week) earlier than infants of euglycemic mothers (paired t-test, $\mathrm{p}<0.001$ ).

Cord blood fetuin-A $(783.6 \pm 320.0$ vs $754.8 \pm 281.9 \mu \mathrm{g} /$ $\mathrm{mL}, \mathrm{p}=0.531$ ) concentrations were similar in GDM and euglycemic pregnancies, while IGF-I $(76.6 \pm 27.8$ vs $68.1 \pm 25.1 \mathrm{ng} / \mathrm{mL}, \mathrm{p}=0.008)$ and IGF-II $(195.3 \pm 32.5$ vs $187.5 \pm 30.8 \mathrm{ng} / \mathrm{mL}, \mathrm{p}=0.042)$ concentrations were significantly higher in GDM pregnancies (table 2 ).

Table 3 presents the partial correlations of cord blood fetuin-A with fetal growth and fetal growth factors in GDM and euglycemic pregnancies adjusting for gestational age at birth/blood sampling. Higher cord blood fetuin-A concentrations were correlated with lower birth length z scores $(\mathrm{r}=-0.24, \mathrm{p}=0.005)$ in GDM pregnancies, while there was no correlation in euglycemic pregnancies. Similarly, higher cord blood fetuin-A levels were correlated with lower birthweight $\mathrm{z}$ scores $(\mathrm{r}=-0.19$, $\mathrm{p}=0.025$ ) in GDM pregnancies, but there was no correlation in euglycemic pregnancies. There were no significant correlations of cord blood fetuin-A with insulin, IGF-I and IGF-II.

Adjusting for maternal and neonatal characteristics (table 4), cord blood fetuin-A concentration remained negatively associated with birth length $\mathrm{z}$ score in GDM pregnancies $(\beta=-0.52, \quad \mathrm{p}=0.012)$, while the association was in the reverse direction and non-significant in euglycemic pregnancies (regression test for interaction: $\mathrm{p}=0.014$ ). A similar negative association was observed in cord blood fetuin-A with birth weight $\mathrm{z}$ score in GDM 
Table 1 Maternal and neonatal characteristics in a nested matched (1:1) study of the newborns of GDM and controls in the Shanghai Birth Cohort

\begin{tabular}{|c|c|c|c|}
\hline & GDM (n=153) & Control $(n=153)$ & P value* \\
\hline \multicolumn{4}{|l|}{ Mothers } \\
\hline Mother's age (years) & $30.35 \pm 3.67$ & $29.86 \pm 3.37$ & 0.192 \\
\hline$>35$ & $17(11.1)$ & $10(6.5)$ & 0.127 \\
\hline Drinking alcohol & $9(7.1)$ & $14(11.3)$ & 0.796 \\
\hline Smoking & $2(1.3)$ & $1(0.7)$ & 1.000 \\
\hline Family history of diabetes & $25(16.5)$ & $14(9.5)$ & 0.056 \\
\hline Education, university degree & $96(62.8)$ & $92(60.5)$ & 0.749 \\
\hline Primiparous & $124(81.1)$ & $124(81.1)$ & 1.000 \\
\hline Prepregnancy BMI $\left(\mathrm{kg} / \mathrm{m}^{2}\right)$ & $23.59 \pm 4.26$ & $21.59 \pm 2.93$ & $<0.001$ \\
\hline BMI category & & & $<0.001$ \\
\hline$<18.5$ underweight & $10(8.1)$ & $14(11.5)$ & \\
\hline $18.5-24.0$ normal weight & $64(51.6)$ & $86(70.5)$ & \\
\hline 24+ overweight/obese & $50(40.3)$ & $22(18.0)$ & \\
\hline Gestational weight gain (kg) & $11.98 \pm 7.85$ & $15.76 \pm 4.21$ & $<0.001$ \\
\hline z score & $-0.35 \pm 1.03$ & $0.17 \pm 0.85$ & $<0.001$ \\
\hline Caesarean delivery & $76(54.3)$ & $46(33.1)$ & $<0.001$ \\
\hline \multicolumn{4}{|l|}{$75 \mathrm{~g}$ OGTT (24-28 weeks) } \\
\hline \multicolumn{4}{|l|}{ Plasma glucose (mmol/L) } \\
\hline Fasting & $4.81 \pm 0.53$ & $4.41 \pm 0.40$ & $<0.001$ \\
\hline 1 hour & $9.87 \pm 1.60$ & $7.42 \pm 1.37$ & $<0.001$ \\
\hline 2 hour & $8.04 \pm 1.64$ & $6.19 \pm 1.10$ & $<0.001$ \\
\hline Insulin for glucose control & $13(8.5 \%)$ & & \\
\hline \multicolumn{4}{|l|}{ Newborns } \\
\hline Birth weight (g) & $3435.88 \pm 488.73$ & $3354.61 \pm 435.65$ & 0.093 \\
\hline z score & $0.40 \pm 1.16$ & $0.15 \pm 0.98$ & 0.044 \\
\hline Birth length (cm) & $49.89 \pm 1.24$ & $49.72 \pm 1.48$ & 0.298 \\
\hline z score & $0.04 \pm 1.06$ & $-0.10 \pm 1.04$ & 0.280 \\
\hline Sex, male & $83(54.2)$ & $83(54.2)$ & 1.000 \\
\hline GA & $39.14 \pm 1.22$ & $39.33 \pm 1.23$ & $<0.001$ \\
\hline Birth weight for GA & & & 0.216 \\
\hline SGA & $10(6.5)$ & $10(6.5)$ & \\
\hline AGA & $119(77.8)$ & $129(84.3)$ & \\
\hline LGA & $24(15.7)$ & $14(9.2)$ & \\
\hline
\end{tabular}

Data presented are mean \pm SD or $\mathrm{n}(\%)$.

${ }^{*} \mathrm{P}$ values from paired t-tests for continuous variables and $\chi^{2}$ tests for categorical variables (McNemar's $\chi^{2}$ tests for dichotomous variables). $\mathrm{P}$ values in bold, $p<0.05$.

AGA, appropriate for gestational age; BMI, body mass index; GA, gestational age; GDM, gestational diabetes mellitus; LGA, large for gestational age (>90th percentile); OGTT, oral glucose tolerance test; SGA, small for gestational age ( $<10$ th percentile, according to the Chinese sex and GA-specific birthweight standards).

$(\beta=-0.45, p=0.036)$ but not in euglycemic pregnancies (test for interaction: $\mathrm{p}=0.013$ ). Excluding GDM patients with insulin treatment, we found that the associations were similar. These associations between fetuin-A and fetal growth (birth length or weight) were similar with further adjustments for cord blood insulin, IGF-I or IGF-II (data not shown) since they were not correlated to fetuin-A.

\section{DISCUSSION}

\section{Main findings}

Our data showed that GDM was not associated with cord blood fetuin-A concentration. The study is the first to demonstrate that cord blood fetuin-A was negatively correlated to fetal growth in GDM pregnancies but not in euglycemic pregnancies. 
Table 2 Cord blood concentrations of fetuin-A and fetal growth factors in the newborns of GDM and controls

\begin{tabular}{|c|c|c|c|}
\hline Cord blood & GDM $(n=153)$ & Control $(n=153)$ & P value* \\
\hline \multirow[t]{2}{*}{ Fetuin-A ( $\mu \mathrm{g} / \mathrm{mL})$} & $783.6 \pm 320.0$ & $754.8 \pm 281.9$ & 0.531 \\
\hline & $719.1(527.7-962.0)$ & $671.3(555.8-974.7)$ & \\
\hline \multirow[t]{2}{*}{ Insulin (pmol/L) } & $36.3 \pm 43.1$ & $31.7 \pm 33.3$ & 0.841 \\
\hline & $22.1(8.9-45.5)$ & $22.4(11.9-39.2)$ & \\
\hline \multirow[t]{2}{*}{ IGF-I (ng/mL) } & $76.6 \pm 27.8$ & $68.1 \pm 25.1$ & 0.008 \\
\hline & $76.3(56.8-89.7)$ & $65.7(48.9-80.9)$ & \\
\hline \multirow[t]{2}{*}{ IGF-II (ng/mL) } & $195.3 \pm 32.5$ & $187.5 \pm 30.8$ & 0.042 \\
\hline & $195.1(171.6-220.1)$ & $188.6(164.8-211.1)$ & \\
\hline
\end{tabular}

Data presented are mean \pm SD and median (IQR). Fetuin-A and IGF-II were measured in cord blood EDTA plasma; insulin and IGF-I were measured in cord blood serum.

${ }^{*} P$ values from paired t-tests in log-transformed biomarker data between the two groups. $P$ values in bold, $p<0.05$.

GDM, gestational diabetes mellitus; IGF, insulin-like growth factor.

\section{Data interpretation and comparisons with previous studies}

We are aware of only one previous study on cord blood fetuin-A in GDM: a small study (12 GDM pregnancies, 12 controls) reported lower cord blood fetuin-A levels in GDM pregnancies. ${ }^{22}$ In contrast, our study in a large cohort (153 GDM pregnancies, 153 controls) showed similar cord blood fetuin-A concentrations between GDM and euglycemic pregnancies, suggesting that the previously reported difference might be a chance finding due to small sample size. As expected, we observed elevated cord blood IGF-I and IGF-II levels in GDM pregnancies, which may well explain the enhanced fetal growth. Unexpectedly, we did not detect a significant difference in cord blood insulin concentrations between the newborns of GDM pregnancies and controls. This could be a consequence of well-managed hyperglycemia in GDM in tertiary obstetric care centers; most women with GDM might have reached euglycemia at late gestation, while cord blood insulin levels might only reflect very shortterm glycemic status. Most notably, average gestational weight gain $\mathrm{z}$ score was lower in GDM versus control groups, suggesting well management of hyperglycemia

Table 3 Partial correlations ${ }^{*}$ of cord blood fetuin-A with fetal growth (birth weight and birth length) and fetal growth factors $(n=306)$

\begin{tabular}{lrrrl}
\hline & \multicolumn{4}{l}{ Cord blood fetuin-A } \\
\cline { 2 - 5 } & GDM & \multicolumn{3}{l}{ Control } \\
& $\mathbf{R}$ & $\mathbf{P}$ & $\mathbf{R}$ & $\mathbf{P}$ \\
\hline Birth weight z score & -0.19 & $\mathbf{0 . 0 2 5}$ & 0.10 & 0.246 \\
Birth length z score & -0.24 & $\mathbf{0 . 0 0 5}$ & 0.06 & 0.479 \\
Insulin & 0.05 & 0.540 & 0.05 & 0.566 \\
IGF-I & -0.03 & 0.754 & 0.07 & 0.416 \\
IGF-II & 0.14 & 0.084 & -0.01 & 0.863 \\
\hline
\end{tabular}

*Data presented are partial correlation coefficients adjusting for gestational age at delivery/cord blood sampling. $P$ values in bold, $\mathrm{p}<0.05$.

GDM, gestational diabetes mellitus; IGF, insulin-like growth factor. in GDM. Insulin, IGF-I and IGF-II are essential for fetal growth. ${ }^{23}$ We did not find any significant correlations of cord blood fetuin-A with insulin, IGF-I and IGF-II, suggesting that any impact of fetuin-A on fetal growth in GDM might operate through pathways independent of these fetal growth factors.

We observed negative correlations of cord blood fetuin-A with birth length and birth weight in the GDM group only. Animal knockout studies have suggested that fetuin-A is essential for longitudinal bone growth. ${ }^{17} 24$ However, fetuin-A may also act as a calcification inhibitor, ${ }^{25} 26$ which might explain the negative correlation of cord blood fetuin-A with linear growth in GDM. Our finding is consistent with the study of Kalabay and colleagues reporting a negative correlation between maternal blood fetuin-A and birth length in GDM pregnancies, ${ }^{9}$ although it is unclear whether maternal fetuin-A could pass the placental barrier. The absence of association in euglycemic pregnancies in our data suggests that under normal physiological conditions, fetuin-A might not affect fetal growth.

The mechanisms driving the conditional association of fetuin-A with fetal growth in GDM are unclear. We speculated that fetuin-A's activity might be modified under the GDM-specific intrauterine endocrine conditions (figure 2). As there are numerous changes in the fetal endocrine environment in GDM, it is possible that some of these changes may potentiate the effects of fetuin-A through altering the protein's post-translational modifications and/or signaling activity. ${ }^{27-29}$ Previous studies have reported variations in fetuin-A's phosphorylation or glycosylation levels in growth-restricted and obese individuals. ${ }^{28}{ }^{29}$ It is plausible that fetuin-A's post-translational modifications may be influenced by GDM, which in turn may explain the observed negative association between fetuin-A and fetal growth in GDM. However, this interpretation is highly speculative as we did not have the data on protein post-translational modifications, which may be the direction for future research. 
Table 4 Associations of cord blood fetuin-A with birth length and birthweight $z$ scores in the newborns of GDM and euglycemic (control) mothers

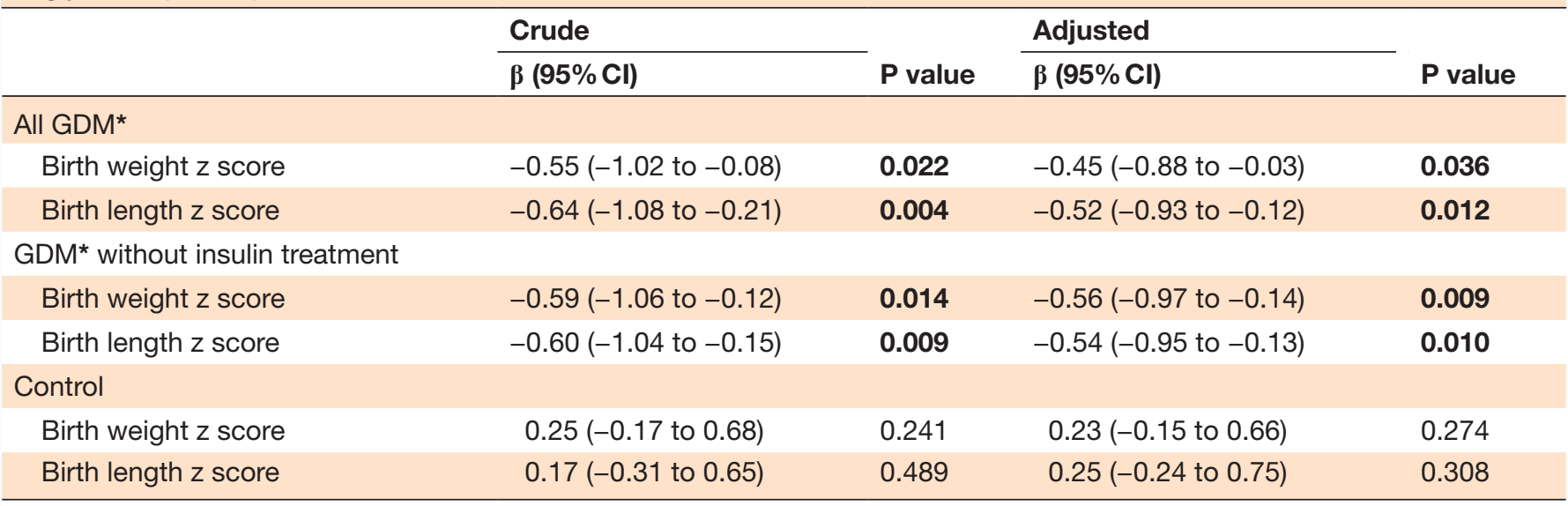

Data $(\beta)$ presented are the changes in fetal growth (birth weight or birth length z score) per natural log unit increment in cord blood fetuin-A concentration from generalized linear models without (crude) or with adjustment for maternal parity, prepregnancy Body Mass Index category, gestational weight gain (z score), family history of diabetes, mode of delivery and gestational age; other maternal and neonatal factors were excluded in the adjusted models since they were non-significant and did not affect the effect estimates. These regression coefficients for fetuin-A in relation to fetal growth were similar in the models with further adjustment for fetal growth factors (insulin, IGF-I or IGF-II; data not shown). $P$ values in bold, $p<0.05$.

${ }^{*}$ There were significant interactions between GDM status and fetuin-A in relation to birthweight $z$ score $(p=0.013)$ or birth length $z$ score $(p=0.014)$.

GDM, gestational diabetes mellitus; IGF, insulin-like growth factor.

\section{Strengths and limitations}

Strengths of our study include the large sample size and high-quality biomarker assays (low intra-assay and interassay coefficients of variation). The main limitation is that we did not have data on fetuin-A isoforms. Future studies may explore whether specific isoforms of fetuin-A may be associated with fetal growth in GDM. Also, our study is observational in nature. Causality could not be affirmed. We could not rule out the possibility of reverse causality. However, similar findings were observed in the analyses with or without adjustment for gestational weight gain, and with or without excluding patients with GDM requiring the use of insulin for hyperglycemia. These sensitivity analyses indicate that the differential
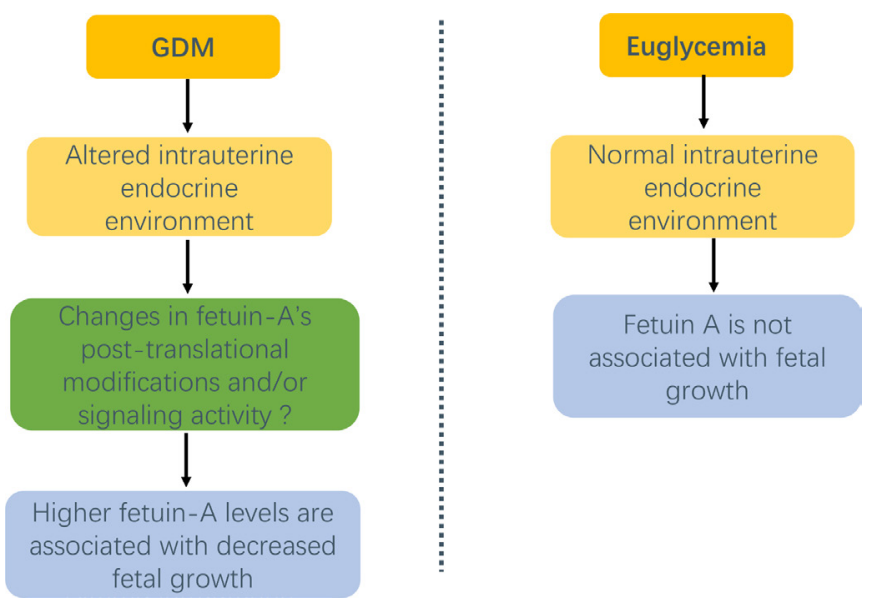

Figure 2 Hypothetical conditional association of fetuin-A with fetal growth in GDM pregnancies. GDM, gestational diabetes mellitus. associations between fetuin-A and fetal growth in GDM versus euglycemic pregnancies are unlikely related to the clinical management of hyperglycemia in pregnancy. The study was based on a Chinese birth cohort. More studies in other populations are warranted to understand the generalizability of the study findings.

In conclusion, our data suggest that GDM is not associated with cord blood fetuin-A level. The study provides the first observation that cord blood fetuin-A levels are negatively correlated to fetal growth in GDM but not in euglycemic pregnancies. This novel finding suggests a GDM-conditional association of fetuin-A with fetal growth and requires confirmation in other independent cohorts.

Author affiliations

${ }^{1}$ Ministry of Education-Shanghai Key Laboratory of Children's Environmental Health, Xinhua Hospital Affiliated to Shanghai Jiaotong University School of Medicine, Shanghai, China

${ }^{2}$ Lunenfeld-Tanenbaum Research Institute, Mount Sinai Hospital, Department of Obstetrics and Gynecology, Institute of Health Policy, Management and Evaluation, Prosserman Center for Population Health Research, Dalla Lana School of Public Health, Faculty of Medicine, University of Toronto, Toronto, Ontario, Canada ${ }^{3}$ Department of Obstetrics and Gynecology, Xinhua Hospital Affiliated to Shanghai Jiaotong University School of Medicine, Shanghai, China

${ }^{4}$ Department of Clinical Assay Laboratory, Xinhua Hospital Affiliated to Shanghai Jiaotong University School of Medicine, Shanghai, China

${ }^{5}$ Department of Pediatrics, Xinhua Hospital Affiliated to Shanghai Jiaotong University School of Medicine, Shanghai, China

Acknowledgements We gratefully acknowledge all research staff who had contributed to patient recruitment and data collection in the Shanghai Birth Cohort.

Contributors Z-CL, LZ, JZ and FO conceived the study with inputs from all coauthors. W-JW, LZ, TZ, G-HZ, KD, M-NY, HH, WW, JZ, FO and Z-CL contributed to the acquisition of research data. W-JW, LZ and SW conducted the literature review and data analysis and drafted the manuscript. All authors contributed in revising the article critically for important intellectual content and approved the final version 
for publication. W-JW and LZ contributed equally. Z-CL was the guarantor of this work, had full access to all the data in the study, and took responsibility for the integrity of the data and the accuracy of the data analysis.

Funding This work was supported by research grants from the Ministry of Science and Technology of China National Key Research and Development Program (2019YFA0802501), the National Natural Science Foundation of China (81571451 and 81673178), the Canadian Institutes of Health Research (158616), the Shanghai Municipal Health and Family Planning Commission (GWIII-26.2) and the National Human Genetic Resources Sharing Service Platform (2005DKA21300). The funders had no role in all aspects of the study, including study design, data collection and analysis, the preparation of the manuscript and the decision for publication.

Competing interests None declared.

Patient consent for publication Not required.

Ethics approval The project was approved by the research ethics committees of the coordination center (Xinhua Hospital, reference number M2013-010) and all participating hospitals. Written informed consent was obtained from all study participants.

Provenance and peer review Not commissioned; externally peer reviewed.

Data availability statement Data are available upon reasonable request. Access to the deidentified participant research data must be approved by the research ethics board on a case-by-case basis, please contact the corresponding author ( zcluo@lunenfeld.ca; ouyangfengxiu@xinhuamed.com.cn) for assistance in data access request.

Open access This is an open access article distributed in accordance with the Creative Commons Attribution Non Commercial (CC BY-NC 4.0) license, which permits others to distribute, remix, adapt, build upon this work non-commercially, and license their derivative works on different terms, provided the original work is properly cited, appropriate credit is given, any changes made indicated, and the use is non-commercial. See: http://creativecommons.org/licenses/by-nc/4.0/.

ORCID iD

Zhong-Cheng Luo http://orcid.org/0000-0002-1794-1312

\section{REFERENCES}

1 Stefan N, Fritsche A, Weikert C, et al. Plasma fetuin-A levels and the risk of type 2 diabetes. Diabetes 2008;57:2762-7.

$2 \mathrm{Ix} \mathrm{JH}$, Wassel CL, Kanaya AM, et al. Fetuin-A and incident diabetes mellitus in older persons. JAMA 2008;300:182-8.

3 Shim YS, Kang MJ, Oh YJ, et al. Fetuin-A as an alternative marker for insulin resistance and cardiovascular risk in prepubertal children. $J$ Atheroscler Thromb 2017;24:1031-8.

4 Mathews ST, Chellam N, Srinivas PR, et al. Alpha2-HSG, a specific inhibitor of insulin receptor autophosphorylation, interacts with the insulin receptor. Mol Cell Endocrinol 2000;164:87-98.

5 Auberger P, Falquerho L, Contreres JO, et al. Characterization of a natural inhibitor of the insulin receptor tyrosine kinase: cDNA cloning, purification, and anti-mitogenic activity. Cell 1989;58:631-40.

6 Pal D, Dasgupta S, Kundu R, et al. Fetuin-A acts as an endogenous ligand of TLR4 to promote lipid-induced insulin resistance. Nat Med 2012;18:1279-85.

7 Chamberlain JJ, Rhinehart AS, Shaefer CF, et al. Diagnosis and management of diabetes: synopsis of the 2016 American diabetes association standards of medical care in diabetes. Ann Intern Med 2016;164:542-52.

8 lyidir OT, Degertekin CK, Yilmaz BA, et al. Serum levels of fetuin a are increased in women with gestational diabetes mellitus. Arch Gynecol Obstet 2015;291:933-7.

9 Kalabay L, Cseh K, Pajor A, et al. Correlation of maternal serum fetuin/alpha2-HS-glycoprotein concentration with maternal insulin resistance and anthropometric parameters of neonates in normal pregnancy and gestational diabetes. Eur $\mathrm{J}$ Endocrinol 2002;147:243-8.

10 Kansu-Celik H, Ozgu-Erdinc AS, Kisa B, et al. Prediction of gestational diabetes mellitus in the first trimester: comparison of maternal fetuin-A, N-terminal proatrial natriuretic peptide, highsensitivity C-reactive protein, and fasting glucose levels. Arch Endocrinol Metab 2019;63:121-7.

11 Monteiro LJ, Norman JE, Rice GE, et al. Fetal programming and gestational diabetes mellitus. Placenta 2016;48:S54-60.

12 Häusler M, Schäfer C, Osterwinter C, et al. The physiologic development of fetuin-a serum concentrations in children. Pediatr Res 2009;66:660-4.

13 Srinivas PR, Wagner AS, Reddy LV, et al. Serum alpha 2-HSglycoprotein is an inhibitor of the human insulin receptor at the tyrosine kinase level. Mol Endocrinol 1993;7:1445-55.

14 Dziegielewska KM, Matthews N, Saunders NR, et al. Alpha 2HSglycoprotein is expressed at high concentration in human fetal plasma and cerebrospinal fluid. Fetal Diagn Ther 1993;8:22-7.

15 Szweras M, Liu D, Partridge EA, et al. Alpha 2-HS glycoprotein/ fetuin, a transforming growth factor-beta/bone morphogenetic protein antagonist, regulates postnatal bone growth and remodeling. J Biol Chem 2002;277:19991-7.

16 Conn KM, Termine JD. Matrix protein profiles in calf bone development. Bone 1985;6:33-6.

17 Seto J, Busse B, Gupta HS, et al. Accelerated growth plate mineralization and foreshortened proximal limb bones in fetuin-A knockout mice. PLoS One 2012;7:e47338.

18 Zhang J, Tian Y, Wang W, et al. Cohort profile: the Shanghai birth cohort. Int J Epidemiol 2019;48:21-21g.

19 Metzger BE, Gabbe SG, Persson B, et al. International association of diabetes and pregnancy study groups recommendations on the diagnosis and classification of hyperglycemia in pregnancy. Diabetes Care 2010;33:e98-82.

20 Zhu L, Zhang R, Zhang S, et al. Chinese neonatal birth weight curve for different gestational age. Zhonghua Er Ke Za Zhi 2015;53:97-103.

21 Chen C, Lu FC, Department of Disease Control Ministry of Health, PR China. The guidelines for prevention and control of overweight and obesity in Chinese adults. Biomed Environ Sci 2004;17:1-36.

22 Šimják P, Cinkajzlová A, Anderlová K, et al. Changes in plasma concentrations and mRNA expression of hepatokines fetuin a, fetuin $\mathrm{B}$ and FGF21 in physiological pregnancy and gestational diabetes mellitus. Physiol Res 2018;67:S531-42.

23 Luo Z-C, Nuyt A-M, Delvin E, et al. Maternal and fetal IGF-I and IGFII levels, fetal growth, and gestational diabetes. J Clin Endocrinol Metab 2012;97:1720-8.

24 Brylka LJ, Köppert S, Babler A, et al. Post-weaning epiphysiolysis causes distal femur dysplasia and foreshortened hindlimbs in fetuinA-deficient mice. PLoS One 2017;12:e0187030.

25 Jahnen-Dechent W, Schäfer C, Heiss A, et al. Systemic inhibition of spontaneous calcification by the serum protein alpha 2-HS glycoprotein/fetuin. Z Kardiol 2001:90:III47-56.

26 Schafer C, Heiss A, Schwarz A, et al. The serum protein alpha 2-Heremans-Schmid glycoprotein/fetuin-A is a systemically acting inhibitor of ectopic calcification. J Clin Invest 2003;112:357-66.

27 Di Cianni G, Miccoli R, Volpe L, et al. Intermediate metabolism in normal pregnancy and in gestational diabetes. Diabetes Metab Res Rev 2003;19:259-70.

28 Karamessinis PM, Malamitsi-Puchner A, Boutsikou T, et al. Marked defects in the expression and glycosylation of alpha2-HS glycoprotein/fetuin-A in plasma from neonates with intrauterine growth restriction: proteomics screening and potential clinical implications. Mol Cell Proteomics 2008;7:591-9.

29 Ren G, Kim T, Papizan JB, et al. Phosphorylation status of fetuin-A is critical for inhibition of insulin action and is correlated with obesity and insulin resistance. Am J Physiol Endocrinol Metab 2019;317:E250-60. 